\title{
Frontières
}

\section{Apocalypse Zombie : l'invasion des morts- vivants dans le roman graphique contemporain}

\section{Antonio Domínguez Leiva}

Volume 25, numéro 2, 2013

Apocalypses et imaginaires de la fin

URI : https://id.erudit.org/iderudit/1024941ar

DOI : https://doi.org/10.7202/1024941ar

Aller au sommaire du numéro

Éditeur(s)

Université du Québec à Montréal

ISSN

1916-0976 (numérique)

Découvrir la revue

Citer cet article

Domínguez Leiva, A. (2013). Apocalypse Zombie : l'invasion des morts- vivants dans le roman graphique contemporain. Frontières, 25(2), 93-108.

https://doi.org/10.7202/1024941ar
Résumé de l'article

La figure du zombie envahit littéralement l'univers de la bande dessinée contemporaine, s'articulant autour de différents mythèmes et motifs qui, à la suite de Romero, en font un héraut inquiétant d'une Nouvelle Apocalypse : la ville en ruines et l'anomie sociale, en proie aux dérèglements de

l' "l'homme-masse " associé au topos fin-de-siècle des " nouveaux barbares " et la régression à la sauvagéité ; la pandémie et le motif de la " rage " associée au cannibalisme des morts ; le triomphe de l'iconographie du cadavre galvanisé ; la surenchère dans le détail gore, à la lisière de l'humour ; la paranoïa envers les appareils idéologiques d'État et notamment les pouvoirs médicaux et militaires ; le sous-texte religieux d'un jugement dernier sans rédemption ; enfin, la « politique » du mauvais goût qui fait des zombies les emblèmes d'une antiesthétique contre-culturelle. 


\section{APOCALYPSE ZOMBIE : \\ L'INVASION DES MORTS- VIVANTS DANS LE ROMAN GRAPHIQUE CONTEMPORAIN}

Antonio Domínguez Leiva, Ph. D. Professeur, Département d'études littéraires, Université du Québec à Montréal

RÉSUMÉ

La figure du zombie envahit littéralement l'univers de la bande dessinée contemporaine, s'articulant autour de différents mythèmes et motifs qui, à la suite de Romero, en font un héraut inquiétant d'une Nouvelle Apocalypse: la ville en ruines et l'anomie sociale, en proie aux dérèglements de l'«l'homme-masse» associé au topos fin-de-siècle des «nouveaux barbares» et la régression à la sauvagéité; la pandémie et le motif de la «rage» associée au cannibalisme des morts; le triomphe de l'iconographie du cadavre galvanisé; la surenchère dans le détail gore, à la lisière de I'humour; la paranoïa envers les appareils idéologiques d'État et notamment les pouvoirs médicaux et militaires; le sous-texte religieux d'un jugement dernier sans rédemption; enfin, la «politique» du mauvais goût qui fait des zombies les emblèmes d'une antiesthétique contre-culturelle.

MOTS-CLÉS: zombies - bande dessinée - abjection - apocalypse esthétique - mort

ABSTRACT

Zombies have litteraly invaded contemporary graphic novels recently, mostly revolving around New Apocalypse motives first established by Romero: destroyed cities, social anomy, human kind's regresssion to barbaric ways; rage like pandemic associated with cannibalism of the dead; the iconographic triomph of glorified cadavers; a visual overkill of gore details which skirt comedy; paranoïa towards state, medical and military powers; an underlying Judgement Day narrative without any hope of redemption; a volontary opposition to cultural aesthetic norms. 
KEYWORDS: zombies - graphic novels - apocalypse - aesthetic death - abjection

Après une éclipse à la fin du siècle passé qui sembla signaler la mort définitive des morts-vivants jusque dans le média filmique qui était devenu leur habitat naturel ${ }^{1}$, ceux-ci ressuscitèrent plus virulents que jamais à l'aube du nouveau millénaire. Ils envahirent alors tous les supports médiatiques de notre sphère globale, des jeux vidéos aux blockbusters, en passant par des sphères jusquelà imperméables à leur charme, telles que la littérature, la philosophie, la théorie économique ou encore, aussi surprenant que cela puisse paraître, les manuels de civilité.

Mais c'est peut-être dans le champ de la bande dessinée que leur irruption massive est la plus frappante. S'il est désormais admis que le film séminal de G. A. Romero Night of the Living Dead (1968) fut nourri par l'iconographie des goules dans les horror comics des années 1950, le zombie fut pratiquement absent du neuvième art pendant que ses congénères envahissaient les écrans du septième, malgré quelques exceptions honorables telles que la surprenante saga Tales of the Zombie, calquée sur le modèle super-héroïque de Swamp Thing. Des tentatives d'acclimatation graphique des modèles cinématographiques telles que l'importante série Deadworld (1987) de Stuart Kerr et Ralph Griffith (publiée deux ans avant l'anthologie littéraire de J. Skipp et $\mathrm{C}$. Spector, Book of the Dead qui préfigurait le phénomène de la «fiction zombie»), rencontrèrent peu d'écho en dehors de la communauté des fans.

Ce n'est qu'au début du XXI siècle que l'œuvre majeure de Robert Kirkman toujours en cours, The Walking Dead (2003- ) lança une véritable zombiemanie graphique à l'image de celle qui sévissait dans les jeux vidéo ou l'industrie filmique, voire la dépassant. Du Remains (2004) de l'enfant terrible Steven Niles, adaptateur par ailleurs de grands classiques zombies tels que I am Legend, Dawn of the Dead ou le 28 days later de Danny Boyle, aux visions post-apocalyptiques de Garth Ennis (Crossed, 2008-) et de Warren Ellis (Blackgas, 2006-7), l'invasion (néo-)zombie est devenue le sous-genre majeur (et le plus rentable) du retour en force des comics d'horreur ${ }^{2}$. La boucle est ainsi bouclée, les zombies étant revenus au média où s'était opéré leur mutation mythopoétique majeure, renouant avec une tradition souterraine qui par deux fois (dans les années 1950 pour les E. C. comics, puis dans les années 1970 pour les Warren comics) flamba avec éclat avant d'être étouffée par la censure.

Le succès des zombies graphiques est tel que le genre même des comics d'horreur rivalise désormais avec la formule jadis hégémonique des sagas de superhéros, ce que confirme ironiquement une œuvre telle que Marvel Zombies, «zombiefication» littérale du panthéon Marvel ou la contrepartie DC tout simplement intitulée Super Zombies. Non contente de rafler le marché américain, la panique néo-zombie est devenue globale, allant de la France (Les Zombies qui ont mangé le monde, 2004-2008, Ma vie de Zombie, 2009) 
à I'Italie (Loving Dead, 2009) et triomphant au sein de l'industrie des mangas où, du Highschool of the Dead (2006) à Tokyo Zombie (2008), elle se fusionne avec des traditions spectrales héritées du shintoïsme et du bouddhisme. L'Apocalypse Zombie est ainsi devenue le cauchemar graphique ultime, synthétisant nos anxiétés culturelles les plus extrêmes.

Ce qui nous frappe le plus au premier abord dans ce raz-de-marée c'est sa totale fidélité à l'iconographie établie il y a un demi-siècle par G. A. Romero, la fixant et la cristallisant au sein de l'imaginaire collectif du Village Global. Dissociant comme l'on sait le zombie de son passé colonial (tout en insérant sa symbolique raciale dans le contexte militant des années 1960), Romero I'inscrit dans les craintes apocalyptiques de I'Amérique à l'âge atomique. Ce brutal aggiornamento accompagnait la modernisation des mythes gothiques traditionnels par la littérature de science-fiction et d'horreur, de Fritz Leiber à Richard Matheson (dont le I am Legend de 1954 préfigure littéralement la saga romérienne), mais aussi et avant tout par les bandes dessinées de la E. C. (Vault of Horror, etc.) et ses nombreuses rivales telles que Black Magic, Dark Mysteries ou l'éphémère Voodoo dont le titre était un clair hommage à I'imaginaire classique du zombie ${ }^{3}$.

La dépossession zombie devint alors le symbole d'une nouvelle époque hantée par l'apocalypse nucléaire, dont la radiation mystérieuse de Night of the Living Dead se fait écho. Éclipsant complètement I'image de l'envoûtement vaudou, la dépersonnalisation zombie rejoint les tropes et les idéologèmes majeurs de la science-fiction et du discours social de la Guerre froide ${ }^{4}$. La trilogie initiale de Romero réinvente ainsi la créature, l'articulant autour de différents mythèmes et motifs qui en feront le héraut inquiétant d'une Nouvelle Apocalypse : la ville en ruines et l'anomie sociale, en proie aux dérèglements de I'«l'homme-masse» associé au topos fin-de-siècle des «nouveaux barbares» et la régression à la sauvagéité; la pandémie et le motif de la «rage» associée au cannibalisme des morts; le triomphe de l'iconographie du cadavre galvanisé ; la surenchère dans le détail gore, à la lisière de l'humour; la paranoïa envers les appareils idéologiques d'État et notamment les pouvoirs médicaux et militaires; le sous-texte religieux d'un jugement dernier sans rédemption; enfin, la «politique» du mauvais goût qui fait des zombies les emblèmes d'une antiesthétique contre-culturelle. Ces éléments régissent encore la construction du «Zombie postmoderne» (Shaviro, 1993, p. 83) qui domine l'invasion graphique en cours, allant parfois jusqu'au simple plagiat ou la pure reprise routinière de clichés renforcée par les impératifs de standardisation industrielle et une certaine pratique de lecture obsessivecompulsive qui caractérisent la pop culture contemporaine.

Réactivée aux lendemains du traumatisme du 11 septembre 2001 et à l'ombre de la pandémie du SARS, nourrie par la rhétorique paranoïaque des années Bush (et sa contrepartie souterraine que sont les théories conspiratives qui de toutes parts prolifèrent), la figure du zombie apocalyptique est devenue le symbole parfait de la Fin des Jours ${ }^{5}$. Dérèglement ultime du corps et de la mort, le néo-zombie est le signe d'un renversement radical du réel; plus que tout autre monstre, il engage le monde à se transformer à son image. 
À la différence du sous-genre de I'holocauste nucléaire, I'apocalypse zombie des comics reste d'ailleurs le plus souvent inexpliquée. Se créent ainsi une perturbation narrative initiale et un phénomène de suspense (plus que la cause de l'invasion, véritable mythe des origines, les survivants recherchent la «cure» ou les moyens d'exterminer à jamais leurs prédateurs), ainsi qu'une ouverture herméneutique du texte à des interprétations symboliques.

Les origines de l'infection sont ainsi inconnues des personnages et des lecteurs de la saga The Walking Dead. Celle-ci commence par la sortie du coma du policier Rick Grimes qui se retrouve dans un hôpital déjà infesté par les morts-vivants. Prisonnier de la focalisation interne de ce personnage, le lecteur va le suivre dans son odyssée personnelle en quête de sa femme et de son enfant, mais aussi de la compréhension de l'événement initial, dont nous trouverons diverses interprétations au fil des rencontres avec les différents survivants. Kirkman fusionne véritablement le sous-genre post-apocalyptique (tel qu'illustré par exemple par la fiction «survivaliste» américaine) avec la tradition zombie, poussée ici jusqu'à des nouveaux niveaux de complexité thématique ${ }^{6}$. Symptomatiquement, au bout des 1000 pages publiées jusqu'à ce jour, nous ne connaissons toujours pas les causes de l'invasion et toutes les tentatives pour comprendre la «transformation » zombie ont eu des résultats catastrophiques (pour le fils de Hershel au chapitre 11 ainsi que pour le zombie attaché par l'assistant du docteur au chapitre 41 , etc. ${ }^{7}$ ).

Zombies! Eclipse of the Undead (2006) commence de même après l'invasion, tandis que Brad, docteur dans le camp de réfugiés du L.A. Memorial Coliseum, évoque les tropes fondateurs du sous-genre: «Nobody knows how it started. Some people say it was radiation from space. Others insist it was a military experiment gone wrong. There are thousand of theories. Does it really matter? [...] The fact is the dead came back... and that they ate the US in a single night» (p. 3). Les lecteurs sont d'emblée plongés dans le drame collectif des survivants qui, en écho évident aux images des lendemains de l'ouragan Katrina, sont abandonnés par les institutions militaires et retournent à une barbarie complète, se tuant et se violant entre eux tandis que les zombies attendent aux portes du camp. Aucune explication du «Z-Day» ne s'ensuivra.

Nous trouvons une variante de ce thème dans Zombie Proof lorsque le serrurier Billy Bob Driwihl rêve d'attaques zombies avant de proposer à ses clients un système de protection à l'épreuve de zombies, dont l'efficacité sera confirmée lors de l'invasion qui peu après s'empare de la petite ville archétypale de San Rosina. L'idée de reconstruire une communauté autarcique, qui est au centre de la Pastorale Américaine dès les jours des Pères Fondateurs, domine ici les autres tropes du genre, dévoilant une des motivations culturelles et psychologiques qui déterminent l'apocalyptisme zombie.

Par opposition aux fictions romériennes classiques où l'invasion zombie marque un événement sans précédent dont on ignore tout du fonctionnement, plusieurs comics se font écho ironique de la saturation médiatique de ses jeunes lecteurs, parfaits connaisseurs des lois du genre. Ainsi dans Living 
with Zombies (M. Billman and C. Hendon, 2004), les personnages principaux (alter egos patents des auteurs) sont des bédéistes accros au massacre de zombies dans les jeux vidéo ${ }^{8}$. «It's just like we've always dreamed, except it's real and I pooped myself », signale Billman lorsque leurs fantasmes deviennent réalité, ce à quoi Chris répond immédiatement: «Really? Fast zombies or the slow ones?», faisant preuve de ses compétences encyclopédiques zombiesques qu'il partage avec son lecteur implicite. Sans se soucier le moindrement de la cause de l'invasion, les personnages l'acceptent comme une nouvelle donnée («I wonder why we aren't zombies», se demande Chris; «I have no clue!», répond Billman, «I just came out of my office and everyone there had turned into the walking dead $\gg(p .5)$, ce qui renforce la composante satirique du American Way of Life déjà si présente dans la trilogie romérienne. Les jeunes bédéistes sont dès lors plus fascinés que terrorisés à la vue de leur premier voisin zombie, devenu spectacle: «Wow, a real life zombie child, held in check, for our very enjoyment, by a chain link fence. I could just stare and chuck rocks at her all day »... Dans ce monde saturé de médias où le réel et le spectacle fusionnent, l'apocalypse zombie elle-même est devenue occasion de consommation: «Oh my god! We're living with zombies! [...] We can finally get an X-box [...]. No one's gonna charge us any money because they are all dead! » (p. 9).

Cette indétermination de la pandémie zombie en fait une parfaite illustration de la «Structure de la menace» étudiée par Luciani Mangin dans la narration hollywoodienne (Mangin, 1990) tout en ouvrant une série de perspectives symboliques, même lorsque l'origine du problème reçoit une quelconque explication. Deadworld débute lorsque le monde plonge dans un holocauste surnaturel d'origine inconnue. Le groupe d'ados de la Louisiane mené par John comprendra, après avoir rencontré un fou égaré nommé Deake, que ce fut une tentative d'ouvrir de nouvelles dimensions qui amena toutes ces créatures dans la leur. Néanmoins l'ouverture de la «porte» a échoué et seulement une partie de l'énergie des morts-vivants a réussi à pénétrer dans ce monde, ce pour quoi les zombies intelligents de cette série, menés par le sinistre King Zombie, sont aux trousses de Deake pour lui faire ouvrir les portes une fois pour toutes. Cette explication surnaturelle de l'invasion s'éloigne de la topique romérienne pour incorporer les tropes de «l'horreur cosmique» définis par Lovecraft et les Mythes de Chtulhu. Or, la plupart des comics restent fidèles au modèle profane mais tout aussi paranoïaque de Romero.

La créature s'articule ainsi à un autre grand thème de la tradition apocalyptique, celui de l'hybris tragique: figure excessive, elle ne peut être que le fruit d'une faute également excessive, souvent associée à une volonté de savoir et/ou de pouvoir déréglée. Militaires (axe du pouvoir) et médecins (axe du savoir) deviennent, dans pratiquement tous les récits graphiques zombies, les causes directes ou indirectes de I'Invasion (notamment dans les réécritures de conflits historiques tels que ceux du Vietnam dans 68 ou de I'Irak dans ZMD Zombies of Mass Destruction) ${ }^{9}$. Le néo-zombie rejoint alors les angoisses d'une «post-humanité» manipulée par les nouveaux pouvoirs médico-disciplinaires qui la configurent. 
Créatures inexplicables et souvent inexpliquées, les zombies conjurent, dans leur pathétisme, l'image d'une fin des temps absurde, parfois provoquée par une pure idiotie, comme c'est le cas du sale gamin qui appuie sur le bouton rouge au début de Remains. Figures de la stérilité, des jardins d'enfance parsemés de carcasses remuantes condensent dans plusieurs de ces œuvres (Blackgas, Crossed, etc.) l'image d'une agénésie désormais sans espoir. Il n'y a nul futur dans ce monde suspendu en proie à l'entropie et la désintégration macabre. L'idée de «tout recommencer», bien qu'elle obsède les survivants de Walking Dead, est sans cesse contestée par les événements, sans qu'aucune solution miraculeuse au style hollywoodien ne soit concevable (ce dont les lettres de fans sont particulièrement reconnaissantes). Même le sousthème adamique propre à la fiction post-apocalyptique est ici problématisé par ce qui devient une sorte de soap zombie: non seulement le couple principal est tiraillé (la fille de Lori n'est pas de Rick mais bien de son rival Shane) mais les familles survivantes sont peu ou prou dysfonctionnelles, entraînant la mort des enfants qui signe la fin de tout espoir (les filles de Hershel décapitées par un psychopathe, etc.). De plus les dénouements sont souvent nihilistes et empreints d'humour noir, comme l'illustre la bombe finale de Blackgas qui, au lieu d'exterminer les masses homicides, finit par répandre le gaz contagieux du titre à toute la planète.

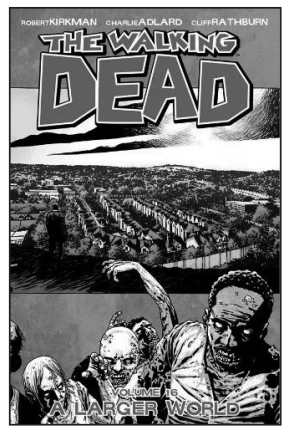

«Peut-être avons-nous mérité ceci», dit un des personnages de Eclipse of the Undead pendant qu'elle observe des figures de hippies qui célèbrent une End of the World Party sur le toit d'un immeuble pendant que tout périclite autour d'eux. La fin de la série célèbre, au contraire, la vie pastorale d'une communauté pacifiée et isolée dans les montagnes, tandis que toutes les villes de la planète sont devenues des «pièges mortels» ( $p .7)$. Cet arc narratif nous dévoile le courant millénariste qui sous-tend le succès contemporain des comics de zombies en un moment de violents fantasmes politiques de purification et de reconstruction du Rêve américain originaire ${ }^{10}$. La pastorale est de fait une des tentations de la fiction post-apocalyptique que I'apocalypse zombie questionne directement ${ }^{11}$. Les waste lands zombies transforment les paysages idylliques de la Pastorale américaine en des Arcadies cancéreuses et polluées, dessinées avec une étrange beauté élégiaque par Tony Moore et Charles Adlard dans Walking Dead, échos mélancoliques de l'écroulement du mythe de la Frontière ${ }^{12}$. De même toutes les tentatives de recréer des espaces pastoraux préservés échouent lamentablement dans la série de Kirkman (la ferme de Hershel, la prison, etc.). Le débat fondateur américain sur les limites entre culture et nature sauvage est clairement tranché par le phénomène zombie où le triomphe de la mort s'accompagne d'un triomphe de la sauvagéité. Le road movie de Rick Grimes se lit ainsi comme descente progressive dans les cercles de l'enfer américain. 
La ville en ruines, icône obsédante des comics de zombies, est le thème corollaire de cet effondrement du mythe arcadien. Correspondance macroscopique du corps déstructuré des masses zombies, la ville apocalyptique n'est plus abandonnée mais grouillante de corps rampants (telle Atlanta dans The Walking Dead, etc.). Littéralement envahies, les métropoles deviennent des charniers, remontant en quelque sorte aux sources mêmes de l'iconographie macabre occidentale, si marquée par l'expérience de la Peste noire. On touche ici à la profonde hantise américaine de la dévastation guerrière «à I'intérieur», curieusement réactivée lors des conflits extérieurs du pays - du Vietnam qui marqua clairement l'œuvre de G. A. Romero et T. Savini à l'invasion de I'Irak qui alimente les comics contemporains.

Cette crainte de l'écroulement de la civilisation - qui est passée de la hantise atomique à celle des attaques biochimiques - est clairement liée à des peurs plus latentes d'anomie sociale. Irrémédiablement collectif (sans quoi il erre, insignifiant, égaré à jamais dans les routes solitaires), le néo-zombie marque la fusion ultime de I'«homme-masse». Monstre par excellence de l'aliénation urbaine, il incarne la dépersonnalisation totale (héritée de l'imaginaire de la Guerre froide) des mégalopoles modernes (Qui ne s'y sent pas entouré de zombies ${ }^{13}$ ). La propre prolifération numérique du zombie-masse transforme l'imaginaire de la transmission vampirique (qui relève plutôt de la contagion vénérienne, donc intime) en cette pandémie rhizomatique que célèbrent sans cesse les couvertures et les planches des comics. Inversement la massification des autres monstres traditionnels (notamment le très individualiste vampire) les rapproche inévitablement du statut zombiesque - c'est le cas, nettement, des hordes vampiriques parties à la recherche du «sang-chair» (blood-meat) dans la saga graphique 30 days of night.

Cette prolifération grouillante, obsessionnellement dépeinte avec le plus grand réalisme par les dessinateurs en quête d'un vertigineux tour de force pictural, permet aussi l'extermination jubilatoire des masses indifférenciées de cadavres (après tout, ne sont-ils pas déjà morts?), héritée directement de I'esthétique des jeux vidéo ${ }^{14}$. Des formes extrêmes de violence telles que l'éviscération, la castration, la décollation ou toute mutilation prolifèrent allègrement dans ces comics, alors qu'elles auraient posé problème dans n'importe quel autre contexte. Il se pourrait d'ailleurs que ces excès gore réactivent une panique morale à l'image de celle qui s'insurgea contre l'explosion de violence graphique qui, sous couvert des tropes classiques du Gothique, se donnait libre cours dans les horror comics. Bien qu'aucune croisade morale ne se soit encore levée contre les zombies graphiques, leur attaque frontale à toute forme de «rectitude politique» en ferait sans nul doute un bouc émissaire de choix.

La violence et la mort règnent sans conteste, dominant les destinées des survivants et des zombies. Prolongeant l'univers tragique décrit par Romero, les personnages de The Walking Dead sont toujours sur le point d'être tués par des zombies, d'autres survivants ou bien des membres de leur propre 
communauté, ce qui les pousse parfois à des mesures désespérées comme le pacte suicidaire des deux adolescents dans le chapitre 14 . Comme le sousgenre «survivaliste» qui triompha pendant les années Reagan et la fin de la Guerre froide ${ }^{15}$, les comics zombies se délectent dans la peinture complaisante de la violence, accumulant les scènes de torture et de viols sadiques. The Walking Dead problématise directement cet imaginaire tout en y ayant recours, avec des épisodes extrêmes tels que le viol de Michonne au chapitre 28, qui, à la limite du soutenable, alimenta un vif débat parmi les lettres des lecteurs, ou encore celui de sa vengeance tout aussi brutale (chapitre 33), selon la logique du sous-genre de rape \& revenge. Dépassant le sadomasochisme macho qui les informe, le scénario de Kirkman reprend la plupart des tropes «survivalistes» tout en questionnant le sens de cette violence qui, bien qu'inévitable dans le contexte de son univers de fiction, reste toujours problématique, dialectique qui fit tout le succès de cette série véritablement «adulte» malgré l'aura d'immaturité profonde qui entoure toujours la figure régressive du zombie.

La masse néo-zombie évoque par ailleurs, de façon tout aussi dérangeante, le fourmillement souterrain d'un sous-prolétariat d'ethnicité douteuse, image déjà présente dans I'œuvre militante de Romero ${ }^{16}$. À la lisière du junkie, du sans-abri et de l'aliéné mental, le zombie devient même l'emblème des nouvelles marginalités (la première planche de Plague of the Living Dead reprend I'image familière des clochards déambulant autour d'un troquet nocturne), auxquelles s'identifient souvent les lecteurs ciblés par le médium des comics, nourri d'une longue tradition contre-culturelle. Comme dans la sous-culture du rock alternatif auquel les comics underground sont directement reliés, une certaine identification empathique rapproche les adolescents de ces outsiders ultimes qui sont avant tout les anges exterminateurs d'une «normalité» adulte méprisée voire haïe. La violence zombie peut ainsi être ambivalente, à la fois subversion défoulatoire de l'ordre social mais aussi affirmation des paniques morales qui en craignent la disparition.

De Plague of the living dead à la saga The Walking Dead, les zombies graphiques parachèvent en réalité tout un imaginaire dystopique (éminemment idéologique) né dans les convulsions révolutionnaires européennes et hanté par le débordement catastrophique de la «populace»; les planches du plus pur splatterpunk de D. Varma, M. Fiumara ou J. Burrows peuvent même facilement évoquer tels délires anthropophagiques des sans-culottes caricaturés par J. Gillray (Le festin cannibale, etc.). Très présente dans la tradition gothique et science-fictionnelle, mais aussi dans la sociologie d'un G. Le Bon, cette vision de la foule dépersonnalisante des déshérités, en proie au délire et prête à toutes les exactions culmine dans les atrocités des masses néozombies. La tradition se superpose ainsi à la perception des problèmes contemporains dans ces comics qui s'abreuvent clairement de l'iconographie des émeutes et riots tels que ceux de Chicago (2001) ou de la Nouvelle Orléans (2005), réactivant en quelque sorte les «Grandes Peurs» de l'Ancien Régime. 
Plusieurs œuvres telles que le Blackgas de W. Ellis ou le Crossed de G. Ennis exploitent explicitement cette symbolique de la psychopathologie collective (provoquée, dans le premier cas, par l'explosion d'un gaz méphitique dans une île isolée et restée inexpliquée dans l'œuvre d'Ennis sans que I'on sache, jusqu'à maintenant, la cause de la folie meurtrière de ses «croisés» - au sens littéral). Cette folie meurtrière investit les espaces sociaux les plus divers, des temples du fast-food (avec les «gags» macabres que l'on peut supposer relatifs à la malbouffe zombie) aux bureaux de poste, les jardins d'enfance ou les maternités: I'image de la Madonne Zombie dans la couverture du quatrième fascicule d'Eclipse of the Undead reste encore une des plus extrêmes dans le répertoire volontiers outrancier des provocations du genre. Faisant du zombisme une véritable hystérie meurtrière (au détriment même de l'iconographie du cadavre revenu à la vie), ces œuvres soulignent la complicité profonde que le mythe entretient avec la hantise des masses criminelles ${ }^{17}$.

Indissolublement lié à cette crainte, le mythe décadent des «nouveaux barbares» partis à l'assaut d'une civilisation périmée active l'image d'une véritable régression à la sauvagéité première. Le néo-zombie est bien un «primitif», et en cela il a gardé ses origines coloniales. Ironiquement, ce sont des Aryens blonds qui dans Crossed reviennent à un état hobbesien où, couverts de quelques cache-sexes faits en peaux humaines (parfois même des visages souffrants) et tenus par des fils électriques, ils érigent en trophées des têtes coupées et violent collectivement des agonisants. Nouveaux Vikings sortis d'un cauchemar aryaniste (c'était le thème d'une autre grande dystopie des années 1970, le Rêve de Fer de N. Spinrad), ils siègent au milieu des lambeaux de cadavres pendus aux lampadaires ou cloués sur les parcomètres.

Le festin cannibale est la culmination de ce réseau symbolique. L'on sait que ce fut le trait le plus marquant de la transformation romérienne du monstre, associé à celui de l'épidémie. Comme le souligne P. Dendle, «Romero libéra le zombie des fers de son maître et l'investit (...) non d'une fonction mais d'une pulsion (manger la chair)» (Dendle, 2000, p. 6). Directement héritière des mutants de $I$ am Legend et du folklore des «goules» des horror comics classiques, cette pulsion orale transforme l'érotique vampirique de la succion (liée, selon la psychanalyse, au stade oral précoce ou pré-ambivalent) en appétit désordonné de la chair humaine, emblème du stade oral-cannibalique ou sadique oral ${ }^{18}$. Le spectateur et le lecteur régressent ainsi avec le zombie à des stades archaïques de leur personnalité, réactivant de façon ambiguë les doubles fantasmes de la destruction de l'objet et du propre sujet. Manger ou/ et être mangé, voilà le dilemme du stade oral que retrouvent étrangement les superhéros décédés de Marvel Zombies et que nous revivons, sous forme de distanciation jouissive, lorsque nous les lisons (Picard, 1986).

Cette oralité déréglée qui se traduit par une «faim» obsessionnelle (comme le soulignent ces zombies parlants que sont les Marvel Zombies ou, plus rudimentaires, ceux de Remains) marque comme un double retour du refoulé, celui, psychique, du stade sadique oral qui détruit tout l'édifice du «Moi» (d'où l'importance de la désagrégation psychique du zombie, no-brainer qui 
est aussi le «non-Moi» absolu), et celui, social, du débordement d'un Quart Monde famélique et ensauvagé, jusqu'alors conjuré et exorcisé par la mythologie de l'hyperconsommation occidentale. Signalant le point absolu de la régression «primitiviste» (écho vivant de l'imaginaire colonial qui avait marqué le premier zombisme et qui, symptomatiquement, s'exprime aussi dans le genre jumeau des «films de cannibales» italiens), l'anthropophagie posthume détruit tout lien social - dans Pieces for Mom (S. Niles, 2007) la mère veut manger le fils qui, resté vivant, s'en occupe et finit par lui procurer, symboliquement, la tête du Père ${ }^{19}$.

L'anthropophagie zombie invertit ainsi diamétralement l'ingestion rituelle des défunts qui caractérise le cannibalisme humain: transgression ultime, ce n'est plus la communauté des vivants qui se ressource par l'incorporation de ses ennemis ou de ses ancêtres, mais bien celle des morts qui étend sa stérilité sur une planète devenue «Terre Gaste». Cette inversion va au-delà des vieilles hantises relatives à la «mastication des morts» qui conditionnèrent l'émergence du mythe vampirique ${ }^{20}$, devenant l'image ultime de l'apocalypse postmoderne, l'anéantissement final d'une humanité qui se mange ellemême. Il se pourrait bien que cette crainte épouse une certaine mauvaise conscience des pays surdéveloppés envers un Quatrième Monde d'affamés dont l'absence même dans l'iconosphère globale montre sa condition de refoulé collectif. C'est aussi la symbolique archaïque des morts ensauvagés qui renaît dans cette «rage» furieuse qui anime les néo-zombies. Comme le souligne M. Vovelle dans I'introduction à son étude La mort en Occident, l'image des trépassés qui font violence aux vivants prédomine dans la « longue histoire» des mentalités paysannes, marquant des rituels exorcisants et des figures folkloriques telle que la terrible Mesnie Hellequin, tardivement christianisée, déchaînement nocturne d'une armée de revenants ${ }^{21}$.

Les comics reprennent dans ce sens toute l'iconographie macabre occidentale, des morts qui assaillent à coup de lances les vivants (Livre d'Heures flamand, Livre d'Heures à l'usage de Rome, etc.) aux cortèges de défunts qui saisissent les vivants, les traînant à leur perte dans les Triomphes de la Mort (Buffalmacco, P. Brueghel, etc.) littéralement repeints par Max Fiumara dans Blackgas et par Varma dans Escape of the Living Dead (et que sont par ailleurs ces comics sinon de vastes Triomphes macabres?). Ces «morts méchants» qui hantent nos cultures, de la Grèce archaïque à la Galice ibérique, trouvent dans la manie destructrice du néo-zombie leur plus virulente image, ajoutant à sa complexité symbolique. Les formes d'agression débordent d'ailleurs le démembrement et la dévoration, allant jusqu'à la transgression du tabou nécrophile dans le droit fil de l'iconographie de la Mort brutalisant la Jeune Fille ( $H$. Baldung Grien, N. Deutsch, etc.), qui troublait autant G. Bataille dans Les larmes d'Eros (1961). Les morts violent ainsi les vivants dans les images à la fois explicitement hardcore et gore de Blackgas, fonctionnant comme un commentaire ironique à la célèbre définition bataillienne de l'érotisme comme «approbation de la vie jusque dans la mort» (Bataille, 1965, p. 15). Cette exaltation posthume de la libido s'étend même jusqu'aux vivants de façon 
typiquement dionysiaque, transformant les camps de réfugiés d'Eclipse of the Undead en orgies sadiennes où Eros et Thanatos fusionnent.

Le paroxysme de ces Triomphes érotiques de la Mort pervertit par ailleurs des images canoniques de l'apocalyptisme occidental. Les comics de zombie présentent ainsi une sécularisation ambiguë de la résurrection biblique des morts qui doit précéder le jugement dernier eschatologique (Actes 24,15). Par opposition à la résurrection des corps glorieux, incorruptibles et puissants des Justes qui seront saisis par la Seconde Venue du Christ (1 Thessaloniciens 4,15-17), les zombies incarnent ceux des damnés, prostrés dans un état de « honte et tourment éternels» (Daniel 12,2, Matthieu 25,41-46 et Jean 5,29). Car si les Justes jouiront d'une félicité sans borne dans leurs corps intégralement restaurés, les méchants «rechercheront la mort et ne la trouveront point, désireront mourir et la mort les fuira » comme on le lit dans l'Apocalypse $(9,6)$. Comme les damnés, les corps des zombies maintiennent les caractéristiques de leur identité, leur intégrité et leur immortalité tout en étant privés des quatre qualités transcendantales qui distinguent les corps des élus: I'impassibilité, la splendeur ou gloire, l'agilité et la subtilité. Les zombies sont donc soumis à toutes sortes de douleurs, obscurs, lents et irrémédiablement matériels ${ }^{22}$. L'apocalypse zombie est bel et bien un Jugement dernier qui aurait lamentablement échoué, où nulle rédemption ni parousie n'est concevable et où seuls restent les corps pseudo-éternels des damnés ${ }^{23}$.

Tels les habitants de l'Enfer de Dante, les zombies sont voués à la répétition infinie de la punition de leurs péchés, saisis de gloutonnerie et de rage, parfois aussi de grotesques appétits sexuels, parcourant inlassablement les lieux qu'ils occupèrent dans leur ancienne existence, privés de raison, de libre arbitre et de toute possibilité de Salut (Paffenroth, 2006, p. 24). Il y a de fait, comme chez Dante, un aspect tragicomique aux déboires de ces êtres caractéristiques de la génération post-grunge, renvoyant à l'absurde existentialiste qui imprégnait la contre-culture des années 1960 dont Romero s'était fait le chantre. Témoins impuissants de leur transformation, les contaminés de Blackgas (qui sont des sortes de «vivants zombies»), demandent constamment la mort tandis qu'ils ne peuvent s'empêcher de suivre leurs pulsions meurtrières et très explicitement sexuelles: «Stop me, stop me. This isn't me. Kill me», supplie un contaminé qui est en passe de violer le cadavre d'un décapité (Blackgas, 2, p. 13). Le pathétisme peut alors faire de ces créatures des êtres sous-beckettiens, comme c'est le cas dans ce squelette souffrant en silence au coin d'une route solitaire dans le très élégiaque et mélancolique Walking Dead de Kirkman (œuvre qui explore particulièrement ce versant pathétique, envers énigmatique du drame des survivants ${ }^{24}$ ).

Les créatures deviennent alors des reflets inquiétants des humains. Ils en prolongent les traits, constituant l'extension logique de la corruption, l'ignorance, la peur ou l'avarice qui dominent les propres survivants - microcosme de l'idéologie américaine du struggle for life héritée du darwinisme social ${ }^{25}$. «Nous sommes les morts qui marchent» clame Rick Grimes dans un des moments les plus dramatiques de la série de Kirkman (chapitre 24, p. 21), cri 
auquel répond de façon ironique le constat du Gouverneur, qui fait figure de Némésis monstrueuse du héros: «Dans un sens je les admire presque. Ils sont nous $\triangleright$. Prolongeant le «paradoxe mathesonien» du survivant exterminateur de ce que l'on pourrait concevoir comme une «nouvelle humanité», à la limite d'une psychose ambiguë, les humains constituent souvent des «monstres» moraux dont les actes égalent ceux de leurs antagonistes inhumains ou post-humains: au-delà du sadisme individuel de personnages tels que le psychopathe autoproclamé Gouverneur dans The Walking Dead ou de collectifs stigmatisés tels que les gangs latinos dans Eclipse of the Undead, les auteurs de comics insistent sur la dégradation morale des survivants ${ }^{27}$.

C'est d'ailleurs le thème principal de la saga de Kirkman, une dégradation qui pousse les personnages les plus humanistes à accomplir des actes inhumains, à commencer par le héros lui-même (lorsque, par exemple, il tue le chef de la prison au chapitre 19), culminant dans l'éviscération par Tyresee de l'amant puis tueur de sa fille (chapitre 14). L'apocalypse zombie devient ainsi le récit du comportement apocalyptique des survivants, de la descente du Gouverneur dans la folie (avec de clairs échos du Kurtz de Heart of Darkness de Conrad) à la brutalité survivaliste de Rick Grimes ${ }^{28}$. De plus le régime fasciste du Gouverneur à Woodbury, bâti sur une actualisation du panem et circenses romain («You have to keep people busy or otherwise they turn against you», dit-il lorsqu'il explique ses Jeux de gladiateurs zombies), est le reflet obscur de la démocratie instable du groupe de Rick lorsqu'elle tente de construire des normes sociales qui régulent et légitiment les nouveaux et nécessaires actes de sauvagerie que le contexte impose ${ }^{29}$.

Inversement, une certaine évolution se fait parfois jour chez les zombies, fruit d'un darwinisme féroce, dans plusieurs de ces œuvres, singeant de façon grotesque la genèse de l'Humanité : ainsi certains zombies commencent dans Remains à en manger d'autres, moins vivaces («the stronger eat the weaker and get stronger», résume Tori), tandis que les proches de Iron Man qui parviennent à dépecer et manger le Surfer d'Argent acquièrent ses pouvoirs, ce qui leur permet de dévorer tous leurs concurrents (Marvel Zombies, v. 3, p. 13). Ces zombies évolués (tels que le corps d'élite qui entoure King Zombie dans Deadworld) accèdent peu à peu à la parole, voire à l'intelligence dans Remains et plus encore dans la réécriture par Kirkman de ces deux extrêmes axiologiques de l'univers des comic books, le zombie et le superhéros (Marvel Zombies), transgressant la définition classique de la créature dans une œuvre qui reste le plus extrême commentaire sur le déclin de l'imaginaire superimpérialiste américain. Enfin le Sauveur lui-même se voit zombifié dans un processus qui est à la fois évolution ultime de la créature et parodie blasphématoire du processus de résurrection, singulièrement avorté, dans une série qui est sans conteste la plus étonnante de toute cette zombie-manie graphique: le webcomic de Rob Liefeld Zombie Jesus qui a déjà fait quantité de petits, répandant une bonne nouvelle pour le moins ambiguë ${ }^{30}$. Est-ce à dire qu'une nouvelle rédemption, faite cette fois-ci à leur image, est possible pour ces créatures de l'infinie finitude? 


\section{BIBLIOGRAPHIE}

CLUTE J. et P. NICHOLS (1999). Encyclopedia of Science Fiction, Londres, Orbit Books.

DELUMEAU, J. (1983). Le péché et la peur, Paris, Fayard.

DENDLE, P. (2000). Zombie Movie Encyclopedia, Jefferson, N.C. McFarland.

KAY, G. (2008). Zombie Movies: The Ultimate Guide, Chicago, Chicago Review Press.

LAGACHE, D. (1968). Vocabulaire de la Psychanalyse, Paris, PUF.

LECOUTEUX, C. (1999). Chasses fantastiques et cohortes de la nuit au Moyen âge, Paris, Imago.

MANGIN, L. (1990). La grande menace: le cinéma américain face au maccarthysme, Paris, Les Trois Cailloux.

MCINTOSH, S. (dir.) (2008). Zombie Culture, Lanham MD, Scarecrow Press.

PAFFENROTH, K. (2006). Gospel of the Living Dead: George Romero's Visions of Hell on Earth, Waco, Baylor University Press.

PICARD, M. (1986). La lecture comme jeu. Essai sur la littérature, Paris, Éd. de Minuit.

PULLIAM, J. (2006). «The Zombie», dans S. T. Joshi (dir.), Icons of Horror and the Supernatural, Greenwood Press.

SHAVIRO, S. (1993). The Cinematic Body, University of Minnesota Press.

VOVELLE, M. (1983). La mort et I'Occident, Paris, Gallimard.

\section{NOTES}

1 «Blood-and-guts terror had ruled the box office in the early 1980 s, but by 1990 the once thriving horror genre had become completely marginalized. [...] By the mid-1990s fewer new zombie movies were being released than at any point since the late 1940 s; the subgenre had completely stalled» (Kay, 2008, p. 183).

2 Parmi plusieurs autres titres: Escape Of The Living Dead, Plague Of The Living Dead, Blackgas, Xxxombies, ZMD Zombies Of Mass Destruction, Zombie Feast, Zombie Proof, War Of The Undead, Zombie Tales, Living With Zombies, etc.

3 Bien qu'il n'y eut qu'un seul E.C. comic directement dévolu à la figure classique du zombie colonial («Zombie», Crypt of Horror, 19), quatre traitaient des cultes vaudou («Voodoo Death», Tales of the Crypt, 23; «Voodoo Horror», Vault of Horror, 17; «Voodoo Vendetta», id., 15; «Voodoo Vengeance», id., 14) et plusieurs autres présentaient une iconographie para-zombie des «goules», omniprésents dans ces publications, comme en témoigne la couverture de Haunt of Fear, 17 (1953). Symptomatiquement G. W. Romero employa ce terme typiquement E.C. de «ghouls» pour désigner ses créatures lorsqu'il travaillait au scénario de Night of the Living Dead.

4 «During the 1950s, the zombie lost some of its connections with voodoo and the Caribbean and became a character whose lack of volition instead represented Cold War fears about Communism's threatened lack of individuality» (J. Pulliam, 2006, p. 733). Romero a par ailleurs toujours reconnu sa dette envers des films tels que The Earth Dies Screaming (1964). 
5 «More than any other monster, zombies are fully and literally apocalyptic [...] they signal the end of the world as we have known it. » (Paffenroth, 2006, p. 23)

6 On peut par ailleurs situer la fiction zombie comme une variation structurelle autour de I'hypergenre post-apocalyptique où «disaster is seen as being followed by savage barbarism and a bitter struggle for survival, with rape and murder commonplace [...]. There is usually an emotional resonance developed from a tension between loss and gain [...]. Such scenarios tempt us with a kind of life in which the individual controls his or her own destiny and in which moral issues are clear-cut» (J. Clute et P. Nichols, 1999, p. 581).

7 «Nobody asks the reason of all this [...] I would like to know the reason of all this obscenity», confesse l'assistant (41, p. 12), tandis que Hershel se refuse à tuer son fils «until we find a way of helping them [...] We don't know what they think or what they feel. We don't know if it's a disease or the side effects of a chemical weapon [...] and you are killing them so as to "take them out of their misery"» (11, p. 6).

8 «Zombies are the perfect monsters in games [...] relatively easy to kill and yet providing continued frights [...] monsters that can be killed guiltfree» (S. McIntosh, «The Evolution of the Zombie» dans S. McIntosh (dir.), 2008, p. 12). Ces créatures dominent par ailleurs les différents univers du jeu vidéo, des maisons hantées victoriennes de la série Alone in the Dark à I'Allemagne nazie de Wolfenstein ou les stations intergalactiques de System Shock, culminant dans ce sous-genre étonnant «où vous êtes le zombie» (Stubbs the Zombie, Rebel Without a Pulse, 2005).

9 Fusionnant les deux figures, les docteurs militaires de Zombie Simon Garth libèrent la créature à la façon classique du mythe de Frankenstein. Il est intéressant de noter que la question de la légitimité de l'autorité est amplement débattue dans la série de Walking Dead, où beaucoup de personnages contestent le leadership du policier Rick Grimes (notamment Iors de son conflit avec Tyresee, aux chapitres 22 à 24).

10 Ces thèmes fondateurs de l'imaginaire culturel américain ont été notamment étudiés par $\mathrm{R}$. Slotkin dans sa somme encore inégalée Gunfighter Nation : the Myth of the Frontier in Twentieth-century America (Atheneum, 1992).

11 «Often the new world is seen as more peaceful and ordered, more in harmony with Nature, than the bustle and strife of civilization. Such stories are often quasi-utopias in feeling and pastoral in their values» (J. Clute et P. Nichols, 1999, p. 581).

12 La magie de ces images a été précisément retenue pour les affiches de l'adaptation télévisuelle qui fait fureur en ce moment sur HBO. Toute la série peut, dans un sens, se lire comme une variation néobaroque autour du motto classique Et in Arcadia Ego, déchaînant les forces macabres latentes sous le rêve profane de l'extase pastorale.

13 D'où la réappropriation ludique de ces «zombie walks» à la mode où des masses se forment à l'image des invasions filmiques, parodiant en fait l'expérience quotidienne urbaine.

14 Parmi des dizaines d'exemples, voir le chapitre 19 de Walking Dead, particulièrement «exterminatoire».

15 «A very particular kind of male-action story, set in post-holocaust venues where law-and-order has disappeared, and where there is effectively no 
restraint upon the behavior of the hero, who therefore kills before he is killed» (J. Clute et P. Nichols, 1999, p. 1188).

16 Nous ne pouvons, dans les limites de cet article, rendre compte de I'intense débat qui accompagna les sous-entendus raciaux de la violence dans la série des Walking Dead. Soulignons néanmoins que Kirkman s'est toujours montré conscient des problèmes sociaux et raciaux qui entourent le genre, tout en essayant de les concilier avec un récit régi par les décisions de personnages complexes et qui ignore délibérément les impératifs de la «correction politique».

17 «The civilized mind strips back to the savage, the primitive, and the unbound. Kill, eat, repeat», explique I'un des maniaques contaminés de Blackgas, pris sur le vif dans le processus de transformation, voire d'«aliénation» stricto sensu (v. 2, p. 24). Symptomatiquement cette hystérie homicide était le sujet de The Crazies, film de Romero (1973) que la multitude d'analyses de sa saga zombie continue à ignorer, malgré le fait que c'en soit le pendant symétrique. Le remake récent de The Crazies (2010) au sein d'une prolifération de films néo-zombies montre par ailleurs le lien indissoluble entre les foules psychopathiques et les morts-vivants.

18 Lagache, 1968, p. 458. Symptomatiquement, l'enfant «sort» de ce stade lorsqu'il peut parler, et le zombie, en y retournant, perd l'usage de la parole.

19 Un sous-thème symptomatique du zombisme est le «Zombie Momism» dont témoignent Pieces for Mom, Braindead (P. Jackson, 1992) ou Ed and his Dead Mother (J. Wacks, 1993) et qui renvoie à la hantise oralecannibale de l'ingestion par la Mère monstrueuse, inversée et conjurée dans l'alimentation de la Mère par son fils.

20 Titre du célèbre traité scientifique de M. Räft, De masticatione mortuorum in tumulis (1725).

21 Ce «système ancien de la mort» est défini par «la présence universelle, obsédante [...] des morts, ces "doubles" que le dernier moment laisse bien vivants, revendicatifs, agressifs, omniprésents. Doubles ou revenants pérennisent au cœur du Moyen Âge I'une des lectures traditionnelles de I'au-delà, celle qui depuis I'Antiquité fait entourer les vivants et leur monde par le monde des morts, des larves, qu'il faut apaiser en assurant leur transit vers le lieu du repos. À ce schéma horizontal qui fait coexister sur le même plan vivants et morts, les religions du livre, et singulièrement le christianisme, ont substitué la perspective verticale qui, valorisant I'hypothèse du salut, conduit l'âme, suivant ce qu'elle a mérité, au ciel ou en enfer - parfois en purgatoire - et annonce la résurrection des corps au jour du Jugement» (Vovelle, 1983, p. 50). Voir aussi Lecouteux, 1999.

22 Les zombies se situent aussi à la croisée des distinctions classiques théologiques entre resurrectionem corporis, resurrectionem carnis and soma pneumaticon qui divisèrent notamment Protestants et Catholiques (voir Delumeau, 1983). Plusieurs des plus typiques «blagues zombies» évoquent en fait des débats historiques tels que la résurrection des corps mutilés, qui avaient déjà fait les délices satiriques des écrivains baroques tels que Quevedo (Sueño de la Muerte, etc.).

23 Ces sous-textes religieux et symboliques sont directement présentés dans le chapitre 41 de The Walking Dead: «We are surrounded by the proof that the Bible was always wrong », dit la fille de Hershel, à qui celuici répond: «Proof? Depending on the interpretation this could be the proof of God's word [...] This can be the resurrection of the dead [...] the 
seven years of tribulation by which we'll be fortified [...] I wonder why we weren't raptured into Heaven». Encore une fois Kirkman explicite et problématise les thèmes latents du mythe zombie, poussant le genre à des nouveaux niveaux de conscience critique.

24 The Walking Dead, v. 1, p. 12 et p. 26. «Les morts, les pauvres morts», écrivait déjà Baudelaire, «ont de grandes douleurs» (Fleurs du Mal, «Spleen et idéal», LXIX, 1857)

$25 \ll$ What is a smart zombie, other than... a human being, a bestial slave to its appetites that struggles to be more? Or what are we, other than... slightly smart zombies, a tribe of deranged, self-destructive cannibals preying on one another? » (Paffenroth, 2006, p. 7)

26 Chapitre 28, p. 18. Il s'agit, on I'aura reconnu, de la reprise de la célèbre réplique de Peter dans Dawn of the Dead de Romero («They're us, that's all, when there's no more room in hell»). «In the end, Romero is asking what is a smart zombie, other than... a human being, a bestial slave to its appetites that struggles to be more? Or what are we, other than... slightly smart zombies, a tribe of deranged, self-destructive cannibals preying on one another? » (Paffenroth, 2006, p. 7)

27 «The horrific nature of zombies, and, many would say, terrorists, is that they may force us to act as barbarically and impetuously as they do [...]. The various movies play this theme out in different ways, and it is clearly one that has developed a great deal, becoming the predominant theme in the genre. 》 (Paffenroth, 2006, p. 10-11).

28 Bien qu'antithétiques, les deux processus sont clairement parallèles: «He was a good man», dit le Docteur en parlant du Gouverneur, «but he enjoyed this far beyond the necessity of protecting us» (chapitre 29), ce qui est exactement I'accusation que Tyresee fit au préalable contre Rick (chapitre 23). De plus tous deux excusent leurs actions en disant qu'elles sont nécessaires à la cohésion de leurs communautés face à l'adversité.

29 Le débat autour de la peine de mort qui traverse toute la série (chapitres $17,18,24$, etc.) en est l'illustration la plus claire. «We cannot ignore the norms, Rick, we must keep some sort of humanity», proteste Tynessee, ce à quoi Rick répond, en guise typiquement survivalist: «the world has changed and we have to change with it [...]. We are already savages, Tyresee. 》

30 Le webcomic original http://zombiejesus.robliefeld.net/ a été suivi par des imitateurs tels que Sam Miserendino avec son Prince of Pieces (http:// www.princeofpieces.com) ou les vignettes satiriques de http://zeejay. comicgenesis.com/ ainsi que par une quantité de graphistes sur différents supports (T-shirts, etc.). (Tous les liens consultés pour la dernière fois le 24/1/2011). 